\title{
Correction to: Mobile phone data reveals the importance of pre-disaster inter-city social ties for recovery after Hurricane Maria
}

Takahiro Yabe ${ }^{1}$, Satish V. Ukkusuri ${ }^{*}$ and P. Suresh C. Rao ${ }^{1,2}$

* Correspondence: sukkusur@ purdue.edu

The original article can be found online at https://doi.org/10.1007/ s41109-019-0221-5

${ }^{1}$ Lyles School of Civil Engineering, Purdue University, 550 Stadium Mall Drive, West Lafayette, IN, USA

Full list of author information is available at the end of the article

\section{Correction to: Appl Netw Sci}

https://doi.org/10.1007/s41109-019-0221-5

Following publication of the original article (Yabe et al. 2019), the author reported to remove the name from the acknowledgements in the original article.

\section{Author details}

'Lyles School of Civil Engineering, Purdue University, 550 Stadium Mall Drive, West Lafayette, IN, USA. ${ }^{2}$ Department of Agronomy, Purdue University, 915 W. State Street, West Lafayette, IN, USA.

Published online: 09 January 2020

\section{Reference}

Yabe et al (2019) Mobile phone data reveals the importance of pre-disaster inter-city social ties for recovery after Hurricane Maria. Appl Netw Sci 4:98. https://doi.org/10.1007/s41109-019-0221-5 